\title{
A New Kind of Weak Solution of Non-Newtonian Fluid Equation
}

\author{
Huashui Zhan ${ }^{1}$ and Bifen $X u^{2}$ \\ ${ }^{1}$ School of Applied Mathematics, Xiamen University of Technology, Xiamen 361024, China
}

${ }^{2}$ School of Teacher Education, Jimei University, Xiamen 361021, China

Correspondence should be addressed to Huashui Zhan; huashuizhan@163.com

Received 5 April 2017; Accepted 15 June 2017; Published 13 July 2017

Academic Editor: Alberto Fiorenza

Copyright (C) 2017 Huashui Zhan and Bifen Xu. This is an open access article distributed under the Creative Commons Attribution License, which permits unrestricted use, distribution, and reproduction in any medium, provided the original work is properly cited.

If the non-Newtonian fluid equation with a diffusion coefficient is degenerate on the boundary, the weak solution lacks the regularity to define the trace on the boundary. By introducing a new kind of weak solutions, the stability of the solutions is established without any boundary condition.

\section{Introduction and the Main Results}

The quasilinear parabolic equation

$$
\begin{gathered}
\frac{\partial u}{\partial t}-\operatorname{div}\left(a(x)|\nabla u|^{p-2} \nabla u\right)-b_{i}(x) D_{i} u+c(x, t) u \\
=f(x, t), \quad(x, t) \in Q_{T}=\Omega \times(0, T)
\end{gathered}
$$

comes from a host of applied fields such as the theory of non-Newtonian fluid, the study of water infiltration through porous media, and combustion theory; one can refer to [1-4] and the references therein. Here $p>1, D_{i}=\partial / \partial x_{i}, a \in C(\bar{\Omega})$, $\Omega \subset \mathbb{R}^{N}$ is a bounded domain with the appropriately smooth boundary $\partial \Omega$. If $a(x)>c>0$, the equations with the type of (1) have been extensively studied; one can refer to [5-7] and the references therein. If $a(x) \geq 0$, one wants to obtain the well-posedness of the equation; the initial value

$$
u(x, 0)=u_{0}(x), \quad x \in \Omega
$$

is invariably imposed. But the boundary value condition

$$
u(x, t)=0, \quad(x, t) \in \partial \Omega \times(0, T)
$$

may be overdetermined. Yin and Wang [8] made a more important devoting to the problem; they classified the boundary into three parts: the nondegenerate boundary, the weakly degenerate boundary, and the strongly degenerate boundary, by means of a reasonable integral description. The boundary value condition should be supplemented definitely on the nondegenerate boundary and the weakly degenerate boundary. On the strongly degenerate boundary, they formulated a new approach to prescribe the boundary value condition rather than defining the Fichera function as treating the linear case. Moreover, they formulated the boundary value condition on this strongly degenerate boundary in a much weak sense since the regularity of the solution is much weaker near this boundary. In a word, instead of the whole boundary condition (3), only a partial boundary condition

$$
u(x, t)=0, \quad(x, t) \in \Sigma_{p} \times(0, T)
$$

is imposed in [8], where $\Sigma_{p} \subseteq \partial \Omega$.

In our paper, for simplism, we assume that $a(x), b_{i}(x)$, and $c(x, t)$ are $C^{1}$ functions, and

$$
\begin{aligned}
& a(x)>0, \quad x \in \Omega, \\
& a(x)=0, \quad x \in \partial \Omega ;
\end{aligned}
$$

the equation is degenerate on the boundary. In our previous works $[9,10]$, we have shown that such degeneracy may result in the fact that the weak solution of the equation lacks the regularity to define the trace on the boundary. Accordingly, how to construct a suitable function, which is independent of the boundary value condition, to obtain the stability of the weak solutions, becomes formidable. The main aim of the paper is to solve the corresponding problem by introducing a new kind of the weak solutions. 
Definition 1. Function $u(x, t)$ is said to be a weak solution of (1) with the initial value (2), if

$$
\begin{array}{r}
u \in L^{\infty}\left(Q_{T}\right), \\
u_{t} \in L^{2}\left(Q_{T}\right), \\
a(x)|\nabla u|^{p} \in L^{1}\left(Q_{T}\right),
\end{array}
$$

and for any function $g(s) \in C^{1}(\mathbb{R}), g(0)=0, \varphi_{1} \in \overline{C_{0}^{1}(\Omega)}$, $\varphi_{2} \in L^{\infty}\left(0, T ; W_{\text {loc }}^{1, p}(\Omega)\right)$,

$$
\begin{aligned}
& \iint_{Q_{T}}\left[u_{t} g\left(\varphi_{1} \varphi_{2}\right)+a(x)|\nabla u|^{p-2} \nabla u \cdot \nabla g\left(\varphi_{1} \varphi_{2}\right)\right. \\
& \quad+u\left(b_{i x_{i}}(x) g\left(\varphi_{1} \varphi_{2}\right)+b_{i}(x) g_{x_{i}}\left(\varphi_{1} \varphi_{2}\right)\right) \\
& \left.\quad-c(x, t) u g\left(\varphi_{1} \varphi_{2}\right)+f(x, t) g\left(\varphi_{1} \varphi_{2}\right)\right] d x d t \\
& \quad=0 .
\end{aligned}
$$

The initial value is satisfied in the sense of that

$$
\lim _{t \rightarrow 0} \int_{\Omega}\left|u(x, t)-u_{0}(x)\right| d x=0 .
$$

The existence of the solution can be proved in a similar way as that in [8]; we omit the details here. In our paper, we mainly are concerned about the stability of the weak solutions without any boundary value condition.

Theorem 2. Let $u, v$ be two weak solutions of (1) with the initial values $u_{0}(x), v_{0}(x)$, respectively; suppose $p>1$ and

$$
\left|b_{i}(x)\right| \leq c a(x)
$$

If

$$
\int_{\Omega} a^{-(p-1)}(x) d x \leq c
$$

then

$$
\begin{array}{r}
\int_{\Omega}|u(x, t)-v(x, t)| d x \leq c \int_{\Omega}\left|u_{0}(x)-v_{0}(x)\right| d x \\
\text { a.e.t } \in[0, T)
\end{array}
$$

is true without any boundary value condition.

Theorem 3. Let $u, v$ be two nonnegative solutions of (1) with the initial values $u_{0}, v_{0}$, respectively. If $1<p \leq 2$ and

$$
\int_{\Omega} a^{-1 /(p-1)}(x) d x<\infty,
$$

then the stability of the weak solutions is true in the sense of (11).

Let us give a comparison between Theorems 2 and 3. To see that, we specially assume that

$$
\begin{aligned}
\Omega & =B_{R}(0)=\left\{x \in \mathbb{R}^{N}:|x|<R\right\}, \\
a(x) & =(R-|x|)_{+}^{\alpha}, \quad \alpha>0 .
\end{aligned}
$$

Then it is easy to know that if $\alpha<1 /(p-1)$, then condition (10) is satisfied; while $\alpha<p-1$, then condition (12) is true. Thus if $1<p<2$, then

$$
\frac{1}{p-1}>p-1,
$$

which implies that when $R \leq 1, R-|x|<1$,

$$
\int_{\Omega} a^{-(p-1)}(x) d x<\int_{\Omega} a^{-1 /(p-1)}(x) d x .
$$

In this case, Theorem 2 cannot include Theorem 3; Theorem 3 has its independent sense. Certainly, if $p \geq 2$, Theorem 2 has its sole important significance.

At the same time, instead of condition (10) (or (12)), we have the following results in the stability or the local stability.

Theorem 4. Let $u, v$ be two nonnegative solutions of (1) with the initial values $u_{0}, v_{0}$, respectively. If $p>1, a(x)$ satisfies (12), and, for small enough $\lambda>0, u(x)$ and $v(x)$ satisfy that

$$
\begin{aligned}
& \frac{1}{\lambda}\left(\int_{\Omega \backslash \Omega_{\lambda}} a(x)|\nabla u|^{p} d x\right)^{(p-1) / p} \leq c, \\
& \frac{1}{\lambda}\left(\int_{\Omega \backslash \Omega_{\lambda}} a(x)|\nabla v|^{p} d x\right)^{(p-1) / p} \leq c,
\end{aligned}
$$

then stability (11) is true. Here $\Omega_{\lambda}=\{x \in \Omega: a(x)>\lambda\}$.

Theorem 5. Let $u, v$ be two solutions of (1) with the differential initial values $u_{0}(x), v_{0}(x)$, respectively. Then there exists a positive constant $\beta \geq 1$ such that

$$
\begin{aligned}
& \int_{\Omega} a^{\beta}|u(x, t)-v(x, t)|^{2} d x \\
& \quad \leq c \int_{\Omega} a^{\beta}\left|u_{0}(x)-v_{0}(x)\right|^{2} d x .
\end{aligned}
$$

In particular, for any small enough constant $\delta>0$,

$$
\begin{aligned}
& \int_{\Omega_{\delta}}|u(x, t)-v(x, t)|^{2} d x \\
& \quad \leq c \delta^{-\beta} \int_{\Omega}\left|u_{0}(x)-v_{0}(x)\right|^{2} d x .
\end{aligned}
$$

If $u_{0}=v_{0}$, by the arbitrariness of $\delta$, one can see that $u(x, t)=v(x, t)$, a.e. $(x, t) \in Q_{T}$; the uniqueness of the solution is true.

We have used some techniques in [9]. But there are many essential improvements in our paper. The main results of my previous work [9] were established on the assumption of that

$$
\begin{aligned}
& u(x) \leq c d(x), \\
& v(x) \leq c d(x),
\end{aligned}
$$

where $d(x)$ is the distance function from the boundary. Condition (19) is much stronger than the usual homogeneous boundary value condition (3), so the conclusions in [9] are not perfect. But in my new paper, we have introduced the new kind of the weak solutions (Definition 1); also we can establish the stability of the weak solutions without any boundary value condition. 


\section{The Proof of Theorem 2}

For small $\eta>0$, let

$$
\begin{aligned}
& S_{\eta}(s)=\int_{0}^{s} h_{\eta}(\tau) d \tau, \\
& h_{\eta}(s)=\frac{2}{\eta}\left(1-\frac{|s|}{\eta}\right)_{+} .
\end{aligned}
$$

Obviously $h_{\eta}(s) \in C(\mathbb{R})$, and

$$
\begin{aligned}
& h_{\eta}(s) \geq 0, \\
&\left|s h_{\eta}(s)\right| \leq 1, \\
&\left|S_{\eta}(s)\right| \leq 1 ; \\
& \lim _{\eta \rightarrow 0} S_{\eta}(s)=\operatorname{sgn} s, \\
& \lim _{\eta \rightarrow 0} s S_{\eta}^{\prime}(s)=0 .
\end{aligned}
$$

Proof of Theorem 2. Let $u, v$ be two solutions of (1) with the initial values $u_{0}(x), v_{0}(x)$. We can choose $S_{\eta}\left(a^{\beta}(u-v)\right)$ as the test function. Then

$$
\begin{aligned}
& \int_{\Omega} S_{\eta}\left(a^{\beta}(u-v)\right) \frac{\partial(u-v)}{\partial t} d x \\
& +\int_{\Omega} a^{\beta+1}(x)\left(|\nabla u|^{p-2} \nabla u-|\nabla v|^{p-2} \nabla v\right) \\
& \cdot \nabla(u-v) S_{\eta}^{\prime}\left(a^{\beta}(u-v)\right) d x \\
& +\int_{\Omega} a(x)\left(|\nabla u|^{p-2} \nabla u-|\nabla v|^{p-2} \nabla v\right) \\
& +\nabla a^{\beta}(u-v) S_{\eta}^{\prime}\left(a^{\beta}(u-v)\right) d x \\
& +\int_{\Omega} D_{i}\left(b_{i}(x)\right)(u-v) S_{\eta}\left(a^{\beta}(u-v)\right) \\
& +a^{\beta} b_{i}(x)(u-v) \cdot(u-v)_{x_{i}} S_{\eta}^{\prime}\left(a^{\beta}(u-v)\right) d x \\
& +\int_{\Omega} b_{i}(x)(u-v) \\
& +a_{x_{i}}^{\beta}(u-v) S_{\eta}^{\prime}\left(a^{\beta}(u-v)\right) d x \\
& +\int_{\Omega} c(x, t)(u-v) S_{\eta}\left(a^{\beta}(u-v)\right) d x d t \\
& =0 .
\end{aligned}
$$

Thus

$$
\begin{gathered}
\lim _{\eta \rightarrow 0} \int_{\Omega} S_{\eta}\left(a^{\beta}(u-v)\right) \frac{\partial(u-v)}{\partial t} d x=\frac{d}{d t}\|u-v\|_{1}, \\
\int_{\Omega} a^{\beta+1}(x)\left(|\nabla u|^{p-2} \nabla u-|\nabla v|^{p-2} \nabla v\right) \\
\cdot \nabla(u-v) S_{\eta}^{\prime}\left(a^{\beta}(u-v)\right) d x \geq 0 .
\end{gathered}
$$

Since $|\nabla a(x)| \leq c$ in $\Omega$, we have

$$
\begin{aligned}
& \mid \int_{\Omega} a^{\beta}(u-v) S_{\eta}^{\prime}\left(a^{\beta}(u-v)\right)\left(|\nabla u|^{p-2} \nabla u\right. \\
& \left.\quad-|\nabla v|^{p-2} \nabla v\right) d x \mid \\
& \quad=\mid \int_{\left\{\Omega: a^{\beta}|u-v|<\eta\right\}} a^{-(p-1) / p} a^{\beta}(u-v) S_{\eta}^{\prime}\left(a^{\beta}(u-v)\right) \\
& \cdot a^{(p-1) / p}\left(|\nabla u|^{p-2} \nabla u-|\nabla v|^{p-2} \nabla v\right) d x \mid \\
& \quad \leq\left(\int_{\left\{\Omega: a^{\beta}|u-v|<\eta\right\}} \mid a^{-(p-1) / p} a^{\beta}(u-v)\right. \\
& \left.\left.\cdot S_{\eta}^{\prime}\left(a^{\beta}(u-v)\right)\right|^{p} d x\right)^{1 / p}\left(\int_{\left\{\Omega: a^{\beta}|u-v|<\eta\right\}} a(x)\right. \\
& \left.\quad \cdot\left(|\nabla u|^{p}+|\nabla v|^{p}\right) d x\right)^{(p-1) / p} .
\end{aligned}
$$

If $\{x \in \Omega: u-v=0\}$ has 0 measures, since

$$
\int_{\Omega} a^{-(p-1)}(x) d x<\infty
$$

consequently

$$
\begin{aligned}
& \left.\left|\int_{\left\{\Omega: a^{\beta}|u-v|<\eta\right\}}\right| a^{-(p-1) / p} a^{\beta}(u-v) S_{\eta}^{\prime}\left(a^{\beta}(u-v)\right)\right|^{p} d x \mid \\
& \quad \leq c \\
& \lim _{\eta \rightarrow 0}\left(\int_{\left\{\Omega: a^{\beta}|u-v|<\eta\right\}} a(x)\left(|\nabla u|^{p}+|\nabla v|^{p}\right) d x\right)^{(p-1) / p} \\
& \quad=\left(\int_{\{\Omega:|u-v|=0\}} a(x)\left(|\nabla u|^{p}+|\nabla v|^{p}\right) d x\right)^{(p-1) / p} \\
& \quad=0 .
\end{aligned}
$$

If $\{x \in \Omega: u-v=0\}$ has a positive measure, obviously,

$$
\begin{gathered}
\lim _{\eta \rightarrow 0}\left(\int_{\left\{\Omega: a^{\beta}|u-v|<\eta\right\}} \mid a^{-(p-1) / p} a^{\beta}(u-v)\right. \\
\left.\left.\cdot S_{\eta}^{\prime}\left(a^{\beta}(u-v)\right)\right|^{p} d x\right)^{1 / p} \\
=\left(\int_{\{\Omega:|u-v|=0\}} \mid a^{-(p-1) / p} a^{\beta}(u-v)\right. \\
\left.\left.\cdot S_{\eta}^{\prime}\left(a^{\beta}(u-v)\right)\right|^{p} d x\right)^{1 / p}=0 .
\end{gathered}
$$

By (21) and condition (10), using the Lebesgue dominated convergence theorem, in both cases, we have

$$
\begin{aligned}
\lim _{\eta \rightarrow 0} \mid \int_{\Omega} a^{\beta}(u-v) S_{\eta}^{\prime}\left(a^{\beta}(u-v)\right) \\
\cdot\left(|\nabla u|^{p-2} \nabla u-|\nabla v|^{p-2} \nabla v\right) d x \mid=0 .
\end{aligned}
$$


While, by (9), $\left|b_{i}(x)\right| \leq c a(x)$,

$$
\begin{aligned}
& \left|\int_{\Omega} b_{i}(x)(u-v) \cdot a_{x_{i}}^{\beta}(u-v) S_{\eta}^{\prime}\left(a^{\beta}(u-v)\right) d x\right| \\
& \quad \leq \int_{\Omega}\left|b_{i}(x)(u-v)\right| S_{\eta}^{\prime}\left(a^{\beta}(u-v)\right) \\
& \cdot\left|a^{\beta}(u-v) \frac{a_{x_{i}}^{\beta}}{a^{\beta}}\right| d x \leq c \int_{\Omega}\left|S_{\eta}^{\prime}\left(a^{\beta}(u-v)\right)\right| \\
& \cdot a^{\beta}(u-v) \frac{\left|b_{i}(x) \nabla a\right|}{a} d x \leq c \int_{\Omega}\left|S_{\eta}^{\prime}\left(a^{\beta}(u-v)\right)\right| \\
& \cdot a^{\beta}(u-v) d x .
\end{aligned}
$$

Then

$$
\begin{aligned}
& \lim _{\eta \rightarrow 0}\left|\int_{\Omega} b_{i}(x)(u-v) \cdot a_{x_{i}}^{\beta}(u-v) S_{\eta}^{\prime}\left(a^{\beta}(u-v)\right) d x\right| \\
& \quad=0 .
\end{aligned}
$$

Moreover, by $\left|b_{i}(x)\right| \leq c a(x)$,

$$
\begin{aligned}
& \left|\int_{\Omega} b_{i}(x) a^{\beta}(x)(u-v) S_{\eta}^{\prime}\left(a^{\beta}(u-v)\right)(u-v)_{x_{i}} d x\right| \\
& \quad \leq c\left(\int_{\Omega} a(x)\left(|\nabla u|^{p}+|\nabla v|^{p}\right) d x\right)^{1 / p} \\
& \quad \cdot\left(\int_{\Omega}\left|a^{\beta}(x)(u-v) S_{\eta}^{\prime}\left(a^{\beta}(u-v)\right)\right|^{p /(p-1)}\right)^{(p-1) / p} .
\end{aligned}
$$

Therefore, we have

$$
\begin{aligned}
& \lim _{\eta \rightarrow 0} \mid \int_{\Omega} b_{i}(x)(u-v) S_{\eta}^{\prime}\left(a^{\beta}(u-v)\right)(u-v)_{x_{i}} \\
& \cdot a^{\beta}(x) d x \mid=0 . \\
& \lim _{\eta \rightarrow 0} \mid \int_{\Omega} D_{i}\left(b_{i}(x)\right)(u-v) S_{\eta}\left(a^{\beta}(u-v)\right)+\int_{\Omega} c(x, t) \\
& \cdot(u-v) S_{\eta}\left(a^{\beta}(u-v)\right) d x \mid \leq c\|u-v\|_{1} \cdot
\end{aligned}
$$

Now, let $\eta \rightarrow 0$ in (22). Then

$$
\frac{d}{d t}\|u-v\|_{1} \leqslant c\|u-v\|_{1}
$$

It implies that

$$
\int_{\Omega}|u(x, t)-v(x, t)| d x \leqslant c(T) \int_{\Omega}\left|u_{0}-v_{0}\right| d x
$$

$\forall t \in[0, T)$.

Theorem 2 is proved.
Corollary 6. Let $u, v$ be two weak solutions of (1) with the initial values $u_{0}(x), v_{0}(x)$, respectively. If (9) is true and it is supposed that

$$
\begin{aligned}
& \int_{\Omega}|\nabla u|^{p-1} d x \leq c, \\
& \int_{\Omega}|\nabla v|^{p-1} d x \leq c .
\end{aligned}
$$

then the stability

$$
\begin{array}{r}
\int_{\Omega}|u(x, t)-v(x, t)| d x \leq c \int_{\Omega}\left|u_{0}(x)-v_{0}(x)\right| d x \\
\text { a.e.t } \in[0, T)
\end{array}
$$

is true without any boundary value condition.

Proof. If (35) is true, then (30) is true by (21). Thus the corollary can be proved in a similar way as that of Theorem 2

\section{The Proofs of Theorems 3 and 4}

Proof of Theorem 3. By Definition 1 , for any $\varphi_{1} \in \overline{C_{0}^{1}(\Omega)}, \varphi_{2} \in$ $L^{\infty}\left(0, T ; W_{\text {loc }}^{1, p}(\Omega)\right)$, we have

$$
\begin{aligned}
& \iint_{Q_{T}}\left[\frac{\partial(u-v)}{\partial t}\left(\varphi_{1} \varphi_{2}\right)\right. \\
& \quad+a(x)\left(|\nabla u|^{p-2} \nabla u-|\nabla v|^{p-2} \nabla v\right) \cdot \nabla\left(\varphi_{1} \varphi_{2}\right) \\
& \quad+(u-v)\left(b_{i x_{i}}(x)\left(\varphi_{1} \varphi_{2}\right)+b_{i}(x)\left(\varphi_{1} \varphi_{2}\right)_{x_{i}}\right) \\
& \left.\quad-c(x, t)(u-v)\left(\varphi_{1} \varphi_{2}\right)\right] d x d t=0 .
\end{aligned}
$$

For a small positive constant $\lambda>0, \Omega_{\lambda}=\{x \in \Omega: a(x)>$ $\lambda$ \} as before, let

$$
\phi_{\lambda}(x)= \begin{cases}1, & \text { if } x \in \Omega_{\lambda}, \\ \frac{1}{\lambda} a(x), & \text { if } x \in \Omega \backslash \Omega_{\lambda} .\end{cases}
$$

Now, we can choose $\varphi_{1}=\phi_{\lambda}(x), \varphi_{2}=\chi_{[\tau, s]} S_{\eta}(u-v)$, and integrate them over $Q_{T}$; accordingly,

$$
\begin{aligned}
& \int_{\tau}^{s} \int_{\Omega} \phi_{\lambda}(x) S_{\eta}(u-v) \frac{\partial(u-v)}{\partial t} d x d t+\int_{\tau}^{s} \int_{\Omega} \phi_{\lambda}(x) \\
& \cdot a(x)\left(|\nabla u|^{p-2} \nabla u-|\nabla v|^{2} \nabla v\right) \cdot \nabla(u-v) S_{\eta}^{\prime}(u \\
& \quad-v) d x d t+\int_{\tau}^{s} \int_{\Omega} a(x)\left(|\nabla u|^{p-2} \nabla u\right. \\
& \left.\quad-|\nabla v|^{p-2} \nabla v\right) \cdot \nabla \phi_{\lambda}(x) S_{\eta}(u-v) d x d t \\
& \quad+\int_{\tau}^{s} \int_{\Omega}(u-v)\left(b_{i x_{i}}(x) \phi_{\lambda}(x) S_{\eta}(u-v)\right. \\
& \left.+b_{i}(x)\left(\phi_{\lambda}(x) S_{\eta}(u-v)\right)_{x_{i}}\right) d x d t \\
& \quad-\int_{\tau}^{s} \int_{\Omega} c(x, t)(u-v) \phi_{\lambda}(x) S_{\eta}(u-v) d x d t=0 .
\end{aligned}
$$


Clearly,

$$
\begin{aligned}
& \int_{\Omega} \phi_{\lambda}(x) a(x)\left(|\nabla u|^{p-2} \nabla u-|\nabla v|^{p-2} \nabla v\right) \cdot \nabla(u-v) \\
& \cdot S_{\eta}^{\prime}(u-v) d x \geq 0, \\
& \mid \int_{\Omega} a(x)\left(|\nabla u|^{p-2} \nabla u-|\nabla v|^{2} \nabla v\right) \\
& \cdot \nabla \phi_{\lambda}(x) S_{\eta}(u-v) d x \mid \leq \int_{\Omega \mid \Omega_{\lambda}} a(x) \\
& \cdot \mid\left(|\nabla u|^{p-2} \nabla u-|\nabla v|^{2} \nabla v\right) \\
& \cdot \nabla \phi_{\lambda}(x) S_{\eta}(u-v) \mid d x \leq \int_{\Omega \backslash \Omega_{\lambda}} a(x) \\
& \cdot\left|\left(|\nabla u|^{p-2} \nabla u-|\nabla v|^{2} \nabla v\right)\right|\left|\nabla \phi_{\lambda}(x)\right| d x \\
& \quad \leq \frac{c}{\lambda}\left[\int_{\Omega \mid \Omega_{\lambda}} a(x)|\nabla u|^{p-1}|\nabla a| d x\right. \\
& \left.\quad+\int_{\Omega \mid \Omega_{\lambda}} a(x)|\nabla v|^{p-1}|\nabla a| d x\right] .
\end{aligned}
$$

Since $1<p \leq 2,|\nabla a| \leq c$,

$$
\int_{\Omega \backslash \Omega_{\lambda}}|\nabla a|^{p} d x \leq c \lambda \leq c \lambda^{p-1}
$$

then

$$
\begin{aligned}
& \frac{c}{\lambda}\left(\int_{\Omega \mid \Omega_{\lambda}} a(x)|\nabla a|^{p} d x\right)^{1 / p} \\
& \quad \leq \frac{c}{\lambda}\left(\lambda \int_{\Omega \backslash \Omega_{\lambda}}|\nabla a|^{p} d x\right)^{1 / p} \leq c .
\end{aligned}
$$

By (41)-(43), using the Hölder inequality,

$$
\begin{aligned}
& \mid \int_{\Omega} a(x)\left(|\nabla u|^{p-2} \nabla u-|\nabla v|^{2} \nabla v\right) \\
& \cdot \nabla \phi_{\lambda}(x) S_{\eta}(u-v) d x \mid \\
& \quad \leq \frac{c}{\lambda}\left[\int_{\Omega_{\mid \Omega_{\lambda}}} a(x)|\nabla u|^{p-1}|\nabla a| d x\right. \\
& \left.\quad+\int_{\Omega \backslash \Omega_{\lambda}} a(x)|\nabla v|^{p-1}|\nabla a| d x\right] \\
& \quad \leq \frac{c}{\lambda}\left(\int_{\Omega \backslash \Omega_{\lambda}} a|\nabla a|^{p} d x\right)^{1 / p} \\
& \quad \cdot\left(\int_{\Omega \backslash \Omega_{\lambda}} a(x)|\nabla u|^{p} d x\right)^{(p-1) / p} \\
& +\frac{c}{\lambda}\left(\int_{\Omega \mid \Omega_{\lambda}} a(x)|\nabla a|^{p} d x\right)^{1 / p} \\
& \quad \cdot\left(\int_{\Omega \backslash \Omega_{\lambda}} a(x)|\nabla v|^{p} d x\right)^{(p-1) / p}
\end{aligned}
$$

$$
\begin{aligned}
& \leq c\left(\int_{\Omega \backslash \Omega_{\lambda}} a(x)|\nabla u|^{p} d x\right)^{(p-1) / p} \\
& +c\left(\int_{\Omega \backslash \Omega_{\lambda}} a(x)|\nabla v|^{p} d x\right)^{(p-1) / p} .
\end{aligned}
$$

Thus

$$
\begin{gathered}
\lim _{\lambda \rightarrow 0} \mid \int_{\Omega} a(x)\left(|\nabla u|^{p-2} \nabla u-|\nabla v|^{p-2} \nabla v\right) \\
\cdot \nabla \phi_{\lambda}(x) S_{\eta}(u-v) d x \mid=0 .
\end{gathered}
$$

There is one more point that I should touch on is that, by that $\int_{\Omega} a^{-1 /(p-1)}(x) d x<c$, using (21) and the Lebesgue dominated convergence theorem,

$$
\begin{aligned}
& \lim _{\eta \rightarrow 0}\left|\int_{\Omega} \phi_{\lambda} b_{i}(x)(u-v) S_{\eta}^{\prime}(u-v)(u-v)_{x_{i}} d x\right| \\
& \leq \lim _{\eta \rightarrow 0}\left(\int_{\Omega} \mid a^{-1 / p} S_{\eta}^{\prime}(u-v)\right. \\
& \left.\left.\cdot(u-v)\right|^{p /(p-1)} d x\right)^{(p-1) / p}\left(\int _ { \Omega } a ( x ) \left(|\nabla u|^{p}\right.\right. \\
& \left.\left.+|\nabla v|^{p}\right) d x\right)^{1 / p}=0, \\
& \lim _{\lambda \rightarrow 0}\left|\int_{\Omega} \phi_{\lambda x_{i}} b_{i}(x)(u-v) S_{\eta}(u-v) d x\right| \leq \lim _{\lambda \rightarrow 0} \frac{c}{\lambda} \\
& \cdot \int_{\Omega \backslash \Omega_{\lambda}}|\nabla a| d x \leq \lim _{\lambda \rightarrow 0} \frac{c}{\lambda}\left(\int_{\Omega \backslash \Omega_{\lambda}} a(x)\right. \\
& \left.\cdot|\nabla a|^{p} d x\right)^{1 / p} \\
& \cdot\left(\int_{\Omega \backslash \Omega_{\lambda}} a^{-1 /(p-1)}(x) d x\right)^{(p-1) / p}=0, \\
& \text { by (43) and } \int_{\Omega} a^{-1 /(p-1)}(x) d x<c, \text { while } \\
& \quad \lim _{\eta \rightarrow 0}\left|\int_{\Omega}(u-v) b_{i x_{i}}(x) \phi_{\lambda}(x) S_{\eta}(u-v) d x\right| \\
& \leq \int_{\Omega}|u-v| d x
\end{aligned}
$$

is obviously true.

By (46)-(47),

$$
\begin{aligned}
& \lim _{\eta \rightarrow 0} \mid \int_{\Omega}(u-v)\left(b_{i x_{i}}(x) \phi_{\lambda}(x) S_{\eta}(u-v)\right. \\
& \left.\quad+b_{i}(x)\left(\phi_{\lambda}(x) S_{\eta}(u-v)\right)_{x_{i}}\right) d x\left|\leq \int_{\Omega}\right| u \\
& \quad-v \mid d x
\end{aligned}
$$


At last,

$$
\begin{gathered}
\lim _{\eta \rightarrow 0} \lim _{\lambda \rightarrow 0} \int_{\tau}^{s} \int_{\Omega} \phi_{\lambda}(x) S_{\eta}(u-v) \frac{\partial(u-v)}{\partial t} d x d t \\
=\int_{\tau}^{s} \frac{d}{d t}\|u-v\|_{L^{1}(\Omega)} d t .
\end{gathered}
$$

Now, after letting $\lambda \rightarrow 0$, let $\eta \rightarrow 0$ in (37). Then, using (40), (45)-(49), and by the Gronwall inequality, we have

$$
\int_{\Omega}|u(x, t)-v(x, t)| d x \leqslant c \int_{\Omega}\left|u_{0}-v_{0}\right| d x .
$$

Proof of Theorem 4. In the first place, similar to the proof of Theorem 3, we have (39)-(41). There is one more point that we should touch on that since $u(x)$ and $v(x)$ satisfy (16), using the Hölder inequality, we have

$$
\begin{aligned}
& \mid \int_{\Omega} a(x)\left(|\nabla u|^{p-2} \nabla u-|\nabla v|^{2} \nabla v\right) \\
& \cdot \nabla \phi_{\lambda}(x) S_{\eta}(u-v) d x \mid \\
& \quad \leq \frac{c}{\lambda}\left(\int_{\Omega \backslash \Omega_{\lambda}} a|\nabla a|^{p} d x\right)^{1 / p} \\
& \quad \cdot\left(\int_{\Omega \backslash \Omega_{\lambda}} a(x)|\nabla u|^{p} d x\right)^{(p-1) / p} \\
& \quad+\frac{c}{\lambda}\left(\int_{\Omega \backslash \Omega_{\lambda}} a(x)|\nabla a|^{p} d x\right)^{1 / p} \\
& \quad \cdot\left(\int_{\Omega \mid \Omega_{\lambda}} a(x)|\nabla v|^{p} d x\right)^{(p-1) / p} \\
& \quad \leq c\left(\int_{\Omega \backslash \Omega_{\lambda}} a|\nabla a|^{p} d x\right)^{1 / p} \\
& \quad+c\left(\int_{\Omega \backslash \Omega_{\lambda}} a(x)|\nabla a|^{p} d x\right)^{1 / p},
\end{aligned}
$$

which goes to zero as $\lambda \rightarrow 0$ since that $a(x) \in C^{1}(\Omega)$. Thus we have (45) too. Last but not the least, since $\int_{\Omega} a^{-1 /(p-1)}(x) d x<$ $\infty$, similar to the proof of Theorem 3, we have (46)-(49). So, as the proof of Theorem 3, we know that stability (11) is true.

\section{The Local Stability}

Proof of Theorem 5. Let $u, v$ be two solutions of (1) with the initial values $u_{0}(x), v_{0}(x)$, respectively. From the definition of the weak solution, if $g(s)=s$, for any $\varphi_{1} \in \overline{C_{0}^{1}(\Omega)}, \varphi_{2} \in$ $L^{\infty}\left(0, T ; W_{\text {loc }}^{1, p}(\Omega)\right)$,

$$
\begin{aligned}
& \iint_{Q_{T}} \varphi_{1} \varphi_{2} \frac{\partial(u-v)}{\partial t} d x d t=-\iint_{Q_{T}} a(x) \\
& \quad \cdot\left(|\nabla u|^{p-2} \nabla u-|\nabla v|^{p-2} \nabla v\right) \nabla\left(\varphi_{1} \varphi_{2}\right) d x d t
\end{aligned}
$$

$$
\begin{aligned}
& -\iint_{Q_{T}}(u-v) \\
& \cdot\left[b_{i x_{i}}\left(\varphi_{1} \varphi_{2}\right)+b_{i}(x)\left(\varphi_{1} \varphi_{2}\right)_{x_{i}}\right] d x d t \\
& +\iint_{Q_{T}}(u-v) c(x, t)\left(\varphi_{1} \varphi_{2}\right) d x d t .
\end{aligned}
$$

In particular, we choose

$$
\begin{aligned}
& \varphi_{1}=a^{\beta}, \\
& \varphi_{2}=\chi_{[\tau, s]}(u-v),
\end{aligned}
$$

where $\chi_{[\tau, s]}$ is the characteristic function on $[\tau, s]$ and the constant $\beta \geq 1$. Denoting $Q_{\tau s}=\Omega \times[\tau, s]$, then

$$
\begin{aligned}
& \iint_{Q_{\tau s}} a(x)\left(|\nabla u|^{p-2} \nabla u-|\nabla v|^{p-2} \nabla v\right) \\
& \nabla\left[(u-v) a^{\beta}\right] d x d t \\
& \quad=\iint_{Q_{\tau s}} a^{1+\beta}\left(|\nabla u|^{p-2} \nabla u-|\nabla v|^{p-2} \nabla v\right) \\
& \nabla(u-v) d x d t+\iint_{Q_{\tau s}} a(x) \\
& \quad \cdot\left(|\nabla u|^{p-2} \nabla u-|\nabla v|^{p-2} \nabla v\right)(u-v) \nabla a^{\beta} d x d t .
\end{aligned}
$$

Clearly,

$$
\begin{aligned}
& \iint_{Q_{\tau s}} a^{1+\beta}\left(|\nabla u|^{p-2} \nabla u-|\nabla v|^{p-2} \nabla v\right) \nabla(u-v) d x d t \\
& \quad \geq 0 .
\end{aligned}
$$

For the second term on the right-hand side of (54), since $|\nabla a| \leq c$,

$$
\begin{aligned}
& \mid \iint_{Q_{\tau s}}(u-v) a(x) \\
& \cdot\left(|\nabla u|^{p-2} \nabla u-|\nabla v|^{p-2} \nabla v\right) \nabla a^{\beta} d x d t\left|\leq \iint_{Q_{\tau s}}\right| u \\
& \quad-v\left|a(x)\left(|\nabla u|^{p-1}+|\nabla v|^{p-1}\right)\right| \nabla a^{\beta} \mid d x d t \\
& \quad \leq c\left(\int_{\tau}^{s} \int_{\Omega} a(x)\left(|\nabla u|^{p}+|\nabla v|^{p}\right) d x d t\right)^{(p-1) / p} \\
& \quad \cdot\left(\int_{\tau}^{s} \int_{\Omega} a(x)\left|\nabla a^{\beta}\right|^{p}|u-v|^{p} d x d t\right)^{1 / p} \\
& \quad \leq c\left(\int_{\tau}^{s} \int_{\Omega} a(x)\left(|\nabla u|^{p}+|\nabla v|^{p}\right) d x d t\right)^{(p-1) / p} \\
& \quad \cdot\left(\int_{\tau}^{s} \int_{\Omega} a^{1+p(\beta-1)}|u-v|^{p} d x d t\right)^{1 / p} \\
& \quad \leq c\left(\int_{\tau}^{s} \int_{\Omega} a^{1+p(\beta-1)}|u-v|^{p} d x d t\right)^{1 / p} .
\end{aligned}
$$


Now, since $\beta \geq 1$ implies $1+p(\beta-1) \geq \beta$, we have

$$
\begin{aligned}
& \left|\iint_{Q_{\tau s}}(u-v) a\left(|\nabla u|^{p-2} \nabla u-|\nabla v|^{p-2} \nabla v\right) \nabla a^{\beta} d x d t\right| \\
& \quad \leq c\left(\int_{\tau}^{s} \int_{\Omega} a^{\beta}|u-v|^{p} d x d t\right)^{1 / p} .
\end{aligned}
$$

If $p \geq 2$,

$$
\begin{aligned}
& \left(\int_{\tau}^{s} \int_{\Omega} a^{\beta}|u-v|^{p} d x d t\right)^{1 / p} \\
& \quad \leq c\left(\int_{\tau}^{s} \int_{\Omega} a^{\beta}|u-v|^{2} d x d t\right)^{1 / p} .
\end{aligned}
$$

If $1<p<2$, by Hölder inequality,

$$
\begin{aligned}
& \left(\int_{\tau}^{s} \int_{\Omega} a^{\beta}|u-v|^{p} d x d t\right)^{1 / p} \\
& \quad \leq c\left(\int_{\tau}^{s} \int_{\Omega} a^{\beta}|u-v|^{2} d x d t\right)^{1 / 2} .
\end{aligned}
$$

Moreover, by $\beta \geq 1, u, v \in L^{\infty}\left(Q_{T}\right)$, we easily see that

$$
\begin{aligned}
& \left|\iint_{Q_{\tau s}} b_{i}(x)(u-v)\left[(u-v) a^{\beta}\right]_{x_{i}} d x d t\right| \\
& \quad=\mid \iint_{Q_{\tau s}} b_{i}(x)(u-v)^{2} a_{x_{i}}^{\beta} d x d t \\
& \quad+\iint_{Q_{s}} b_{i}(x)(u-v)(u-v)_{x_{i}} a^{\beta} d x d t \mid \\
& \quad \leq c \int_{\tau}^{s} \int_{\Omega_{\lambda}}|u-v| a^{\beta-1} d x \\
& \quad+\left(\int_{\tau}^{s} \int_{\Omega} a^{(\beta-(1 / p)) p^{\prime}}|u-v|^{p^{\prime}} d x d t\right)^{1 / p^{\prime}} \\
& \quad+\left(\int_{\tau}^{s} \int_{\Omega} a(x)\left(|\nabla u|^{p}+|\nabla v|^{p}\right) d x d t\right)^{1 / p} \\
& \quad \leq c\left(\int_{\tau}^{s} \int_{\Omega} a^{\beta}|u-v|^{2} d x d t\right)^{1 / 2} \\
& \quad+c\left(\int_{\tau}^{s} \int_{\Omega} a^{\beta}|u-v|^{2} d x d t\right)^{1 / p^{\prime}} .
\end{aligned}
$$

At last, it is easily to deduce that

$$
\begin{aligned}
& \left|\iint_{Q_{\tau s}}\left(b_{i x_{i}}(x)(u-v)^{2}+c(x, t)(u-v)^{2}\right) a^{\beta} d x d t\right| \\
& \quad \leq c\left(\int_{\tau}^{s} \int_{\Omega} a^{\beta}|u-v|^{2} d x d t\right)^{1 / 2}, \\
& \iint_{Q_{\tau s}}(u-v) a^{\beta} \frac{\partial(u-v)}{\partial t} d x d t
\end{aligned}
$$

$$
\begin{aligned}
= & \int_{\Omega} a^{\beta}[u(x, s)-v(x, s)]^{2} d x \\
& -\int_{\Omega} a^{\beta}[u(x, \tau)-v(x, \tau)]^{2} d x .
\end{aligned}
$$

By (54)-(61), we let $\lambda \rightarrow 0$ in (52). Then

$$
\begin{aligned}
\int_{\Omega} \rho^{\beta} & {[u(x, s)-v(x, s)]^{2} d x } \\
& -\int_{\Omega} a^{\beta}[u(x, \tau)-v(x, \tau)]^{2} d x \\
\leq & c\left(\int_{0}^{s} \int_{\Omega} \rho^{\beta}|u(x, t)-v(x, t)|^{2} d x d t\right)^{q},
\end{aligned}
$$

where $q<1$. By (62), we easily show that

$$
\begin{aligned}
& \int_{\Omega} a^{\beta}|u(x, s)-v(x, s)|^{2} d x \\
& \quad \leq \int_{\Omega} a^{\beta}|u(x, \tau)-v(x, \tau)|^{2} d x .
\end{aligned}
$$

Thus, by the arbitrariness of $\tau$, we have

$$
\int_{\Omega} a^{\beta}|u(x, s)-v(x, s)|^{2} d x \leq \int_{\Omega} a^{\beta}\left|u_{0}-v_{0}\right|^{2} d x .
$$

The proof is complete.

\section{Conflicts of Interest}

The authors declare that they have no conflicts of interest.

\section{Acknowledgments}

The paper is supported by Natural Science Foundation of Fujian Province (no. 2015J01592), supported by Science Foundation of Xiamen University of Technology, China.

\section{References}

[1] R. Aris, The Mathematical Theory of Diffusion and Reaction in Permeable Catalysts, I, II, Clarendon, Oxford, UK, 1975.

[2] E. C. Childs, An Introduction to the Physical Basis of Soil Water Phenomena, Wiley, London, UK, 1969.

[3] Z. Wu, J. Zhao, J. Yin, and F. Li, Nonlinear Diffusion Equations, World Scientific Publishing, Toh Tuck Link, Singapore, 2001.

[4] E. DiBenedetto, Degenerate Parabolic Equations, Springer, New York, NY, USA, 1993.

[5] E. Nabana, "Uniqueness for positive solutions of $p$-Laplacian problem in an annulus," Annales de la Faculté des Sciences de Toulouse, vol. 8, no. 1, pp. 143-154, 1999.

[6] K. Lee, A. Petrosyan, and J. L. Vazquez, "Large-time geometric properties of solutions of the evolution $p$-Laplacian equation," Journal of Differential Equations, vol. 229, no. 2, pp. 389-411, 2006.

[7] J. N. Zhao, "Existence and nonexistence of solutions for $u_{t}$ $\operatorname{div}\left(|\nabla u|^{p-2} \nabla u\right)=f(\nabla u, u, x, t)$, Journal of Mathematical Analysis and Applications, vol. 172, no. 1, pp. 130-146, 1993. 
[8] J. Yin and C. Wang, "Evolutionary weighted $p$-Laplacian with boundary degeneracy," Journal of Differential Equations, vol. 237, no. 2, pp. 421-445, 2007.

[9] H. Zhan, "The stability of the solutions of an equation related to the $p$-Laplacian with degeneracy on the boundary," Boundary Value Problems, vol. 2016, article 178, 2016.

[10] H. Zhan, "The solutions of a hyperbolic-parabolic mixed type equation on half-space domain," Journal of Differential Equations, vol. 259, no. 4, pp. 1449-1481, 2015. 


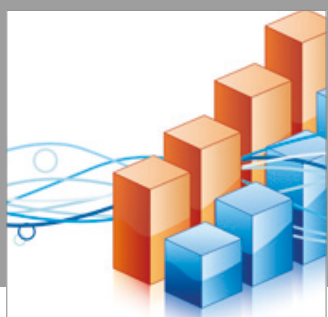

Advances in

Operations Research

vatersals

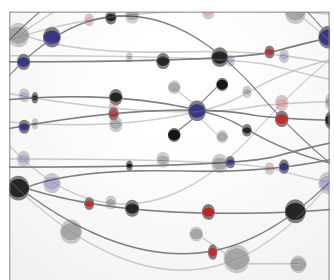

\section{The Scientific} World Journal
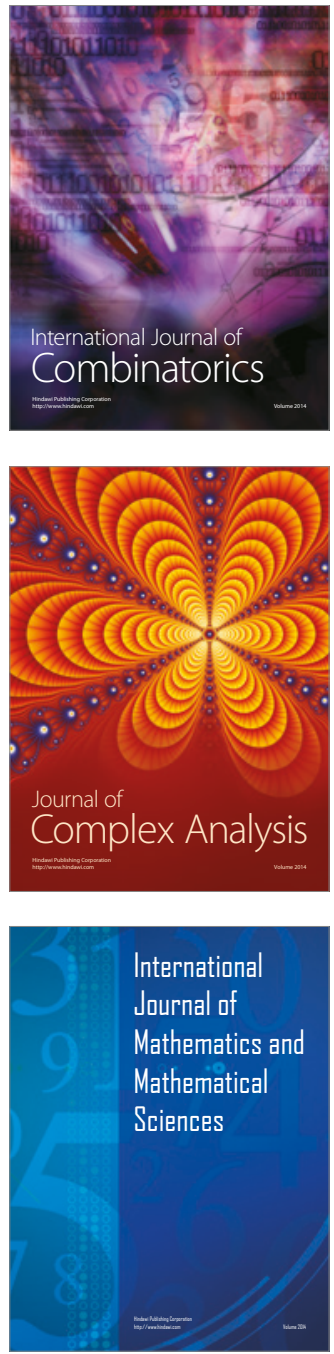
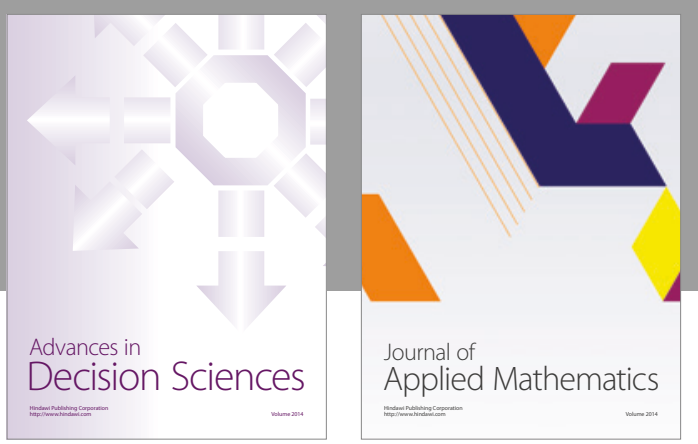

Algebra

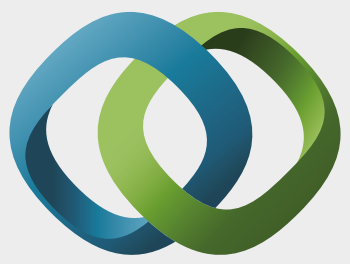

\section{Hindawi}

Submit your manuscripts at

https://www.hindawi.com
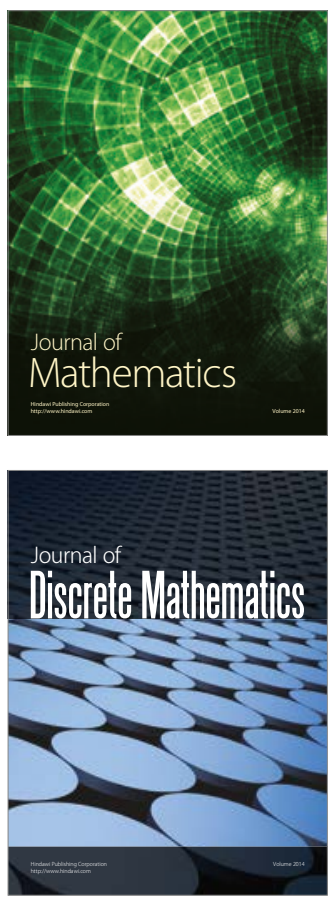

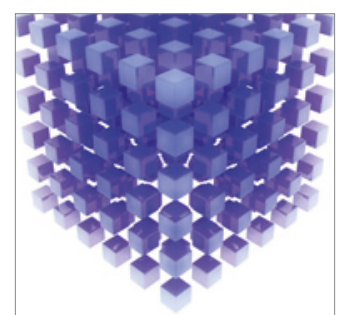

Mathematical Problems in Engineering
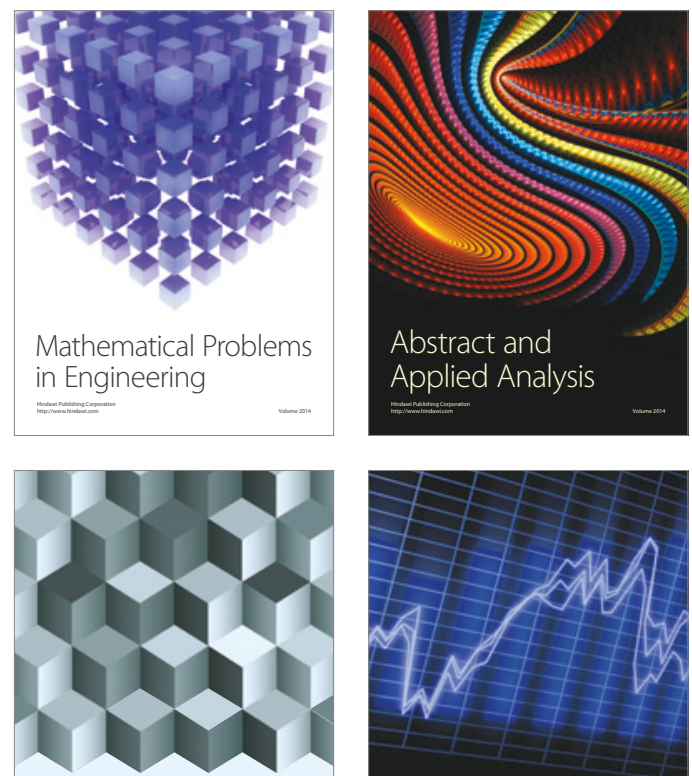

Journal of

Function Spaces

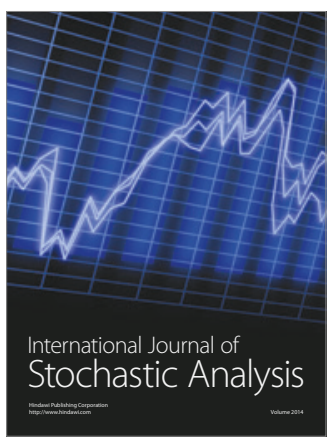

Probability and Statistics
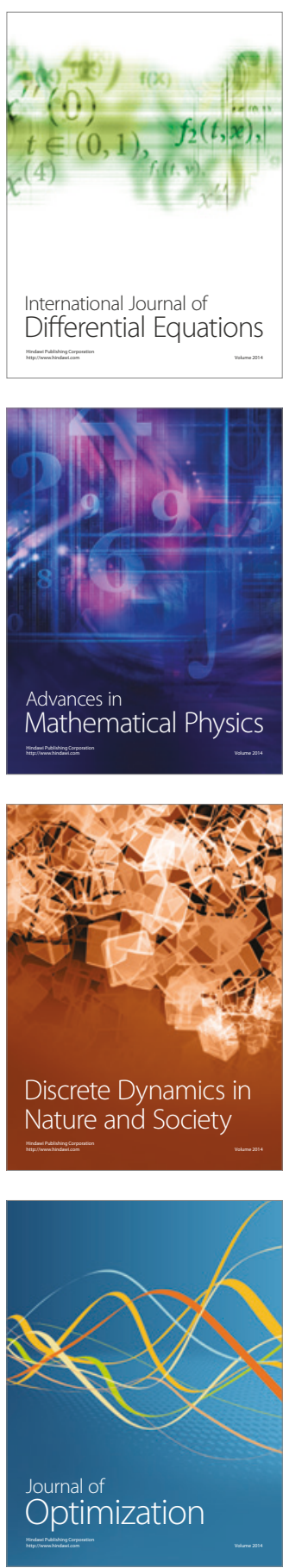